\title{
Evaluation of Serum Magnesium Level along with Lipid Profile in a Gujarati Population diagnosed with Diabetes Mellitus
}

\author{
${ }^{1}$ Sandip S Sendhav, ${ }^{2}$ Amit Kakaiya, ${ }^{3}$ Bijoya Chatterjee
}

\begin{abstract}
Aims and objectives: Diabetes is a common endocrinal disorder. Abnormal lipid and magnesium levels are observed in diabetes in many studies. The current study was done with an aim to find the relationship between lipid with magnesium and diabetes mellitus in Gujarati population.
\end{abstract}

Materials and methods: The cross-sectional study included 60 diabetics and 50 healthy subjects. Each subject was interviewed, examined, and investigated for serum lipid profile and magnesium. A 12-hour overnight fasting was recommended. The statistical analysis of data obtained was done by Student's t-test and calculation of Pearson correlation coefficient.

Result: Routine biochemical investigations showed a significant rise $(p<0.01)$ of fasting plasma glucose, triglycerides (TGs), total cholesterol (TC), low-density lipoprotein-cholesterol (LDL-C), and very low-density lipoprotein-cholesterol (VLDL-C) in diabetics, in comparison with controls $(p<0.01)$. Among diabetics, males have significantly higher $(p<0.01)$ TC, TG, and LDL-C while significantly lower high-density lipoprotein-cholesterol (HDL-C). Significant inverse correlation of magnesium with $\mathrm{TC}(r=-0.18)$, TGs ( $r=-0.14)$, LDL-C ( $r=-0.27)$, fasting blood sugar (FBS; $r=-0.12)$ and direct correlation with HDL-C $(r=026)$ were observed in cases.

Conclusion: The major highlights of the current study are lower magnesium levels in cases compared with controls. In the current study, serum magnesium level has been found to be inversely related to cholesterol, TG, and LDL-C levels, while it is directly associated with HDL-C level. Hence, it could play a role in controlling the risk of coronary artery disease (CAD)associated morbidities in future.

Keywords: Coronary artery disease, Diabetes mellitus, Lipid profile, Magnesium.

How to cite this article: Sendhav SS, Kakaiya A, Chatterjee B. Evaluation of Serum Magnesium Level along with Lipid Profile in a Gujarati Population diagnosed with Diabetes Mellitus. Indian J Med Biochem 2017;21(2):112-116.

Source of Support: Nil

Conflict of interest: None

\footnotetext{
${ }^{1,2}$ Assistant Professor, ${ }^{3}$ Tutor

${ }^{1-3}$ Department of Biochemistry, M. P. Shah Government Medical College, Jamnagar, Gujarat, India
}

Corresponding Author: Amit Kakaiya, Assistant Professor Department of Biochemistry, M. P. Shah Government Medical College, Jamnagar, Gujarat, India, Phone: +918866099116 e-mail: a_kakaiya@yahoo.com

\section{INTRODUCTION}

Diabetes is the most common endocrinal disorder seen in clinical practice. India has already become the "diabetes capital" of the world with over three crore affected patients. Between 1995 and 2025, there is a prediction of a 35\% increase in the worldwide prevalence of diabetes. The rising number of people with diabetes will be mainly in populations of developing countries, leading to more than 300 million people with diabetes globally by 2025 . The disease has tremendous impact on the quality-oflife. Morbidity and mortality occur due to complications that affect the small vessels resulting in retinopathy, nephropathy, and neuropathy and large vessels resulting in ischemic heart disease and stroke. ${ }^{1}$

In diabetes mellitus, lipid abnormalities are almost the rule. Typical findings are elevation of TC and VLDL-C, TG concentration, exaggerated postprandial lipemia, lowering of HDL-C and a predominance of small dense LDL particles. ${ }^{2}$ Triglyceridemia has been associated with increased risk of coronary heart disease both in nondiabetic and diabetic subjects. ${ }^{3,4}$ Remnants of TG-rich lipoproteins seem to be extremely atherogenic. ${ }^{5}$ The LDL-C is related to lifestyle factors, such as diet and exercise. ${ }^{6}$ It has been associated with metabolic syndrome. ${ }^{7}$ The proatherogenic properties of small dense LDL particles may relate to their ability to penetrate the arterial wall, thereby, making them more susceptible to oxidation, and thus, indirectly linked with CAD ${ }^{8,9}$ The CAD represents a wide spectrum of diseases ranging from angina pectoris, myocardial infarction, and sudden death due to silent myocardial ischemia. Silent myocardial ischemia has a reported prevalence of 10 to $20 \%$ in diabetic population as compared with 1 to $4 \%$ in nondiabetic population. ${ }^{10}$

Magnesium is an important intracellular cation, i.e., distributed in three major compartments: Mineral phase of bones $(65 \%)$, intracellular space $(34 \%)$, and extracellular fluid (1\%). About one-third of extracellular magnesium is bound to plasma proteins; the rest is free and presumably biologically available. In several studies, reduced magnesium concentration has been observed in diabetes mellitus. Despite good nutritional status of diabetics, low magnesium level probably results from glycosuria-related hypermagnesemia, nutritional factors, 
Evaluation of Serum Magnesium Level along with Lipid Profile

or hyperinsulinemia. There is a link between hypomagnesemia and reduction of tyrosine kinase activity at the insulin receptor level. This may result in impairment of insulin action and development of insulin resistance. Various evidences suggest that magnesium supplementation could be useful in the treatment of diabetes and to prevent the development of its chronic complications. ${ }^{11}$

Hypomagnesemia can increase platelet reactivity, vascular and adrenal response to angiotensin-II, enhance thromboxane A2 release, and lead to organ damage from free radicals. Magnesium deficiency has also role in perturbation of lipid metabolism, especially in diabetes patients. ${ }^{11}$

Therefore, this study was carried out to compare the magnesium levels in patients with diabetes mellitus with those of healthy controls. It also aimed to explore the correlation between magnesium levels and lipid profile in order to find the CAD risk in such subjects in comparison with healthy controls.

\section{MATERIALS AND METHODS}

This cross-sectional study was carried out in a civil hospital at Ahmedabad, India. It included 60 cases of diabetes mellitus that had come to the diabetic clinic of the hospital and 50 apparently healthy subjects as a control group. The patients were primarily diagnosed by clinical examination and evaluated by investigations. The subjects in the control group were selected from the healthy subjects. The blood samples were drawn from all the patients after a minimum 12 hours of complete fasting. About $5 \mathrm{~mL}$ of blood samples was obtained from cases and control subjects. The blood samples were centrifuged at $5000 \mathrm{rmp}$ for 10 minutes at room temperature within 1 hour of collection. The serum thus obtained was analyzed for FBS by glucose oxidase-peroxidase method, TC by cholesterol oxidase/peroxidase-p-aminophenazone method, TG by glycerol-3-phosphate oxidase/phenol + aminophenazone method, HDL-C and LDL-C by direct enzymatic method, and magnesium by Calmagite method. Student's t-test was used for comparison of the mean values in independent groups. Pearson's correlation coefficients were calculated for the different variables. A p-value of $<0.05$ was considered statistically significant. All statistical analysis was carried out using the software known as Graphpad Prism version 5.

\section{RESULTS}

The age distributions of patients and controls were between 25 and 27 years (Table 1). Out of 60 diabetic patients, 31 were males and 29 were females. Out of 50 controls, 28 were males and 22 were females. The body mass index (BMI) (Table 1) of diabetics was found to be more than the controls. Out of 60 diabetics, 43 had a BMI
Table 1: Demographic characteristic of case and control groups

\begin{tabular}{lll}
\hline & Case & Control \\
\hline Age (years) & & \\
$25-45$ & 29 & 27 \\
$46-65$ & 27 & 22 \\
$>65$ & 4 & 1 \\
Total & 60 & 50 \\
Gender & & \\
Male & 31 & 28 \\
Female & 29 & 22 \\
Total & 60 & 50 \\
Dietary habit & & \\
Veg & 37 & 36 \\
Non-veg & 23 & 14 \\
Total & 60 & 50 \\
BMI $\left(\mathrm{kg} / \mathrm{m}^{2}\right)$ & & \\
18.5-22.9 & 17 & 29 \\
$\geq 23$ & 43 & 21 \\
\hline Total & 60 & 50 \\
\hline
\end{tabular}

greater than $23 \mathrm{~kg} / \mathrm{m}^{2}$ compared with 21 controls out of 50 . The present study shows that all diabetic subjects have significantly higher cholesterol $(p<0.01)$, TG $(p<0.01)$, and LDL-C $(p<0.01)$, while significantly lower HDL-C $(p<0.01)($ Graph 1) and magnesium levels as compared with healthy subjects (Table 2 and Graph 1).

Diabetic males showed a significant increase in cholesterol $(p<0.01)$, TG $(p<0.01)$, and LDL-C $(p<0.01)$, while significantly lower HDL-C $(\mathrm{p}<0.01)$ and magnesium $(\mathrm{p}<0.01)$ as compared with diabetic females (Table 3 and Graph 2). However, no significant differences were observed between genders in the control group. A significant inverse correlation of magnesium with TC $(\mathrm{r}=-0.18)$, TGs $(\mathrm{r}=-0.14)$, LDL-C $(\mathrm{r}=-0.27)$, and FBS $(r=-0.12)$ and a positive correlation with HDL-C $(\mathrm{r}=0.26)$ were observed in cases (Graphs 3 to 6). However, no such difference was observed in control group as shown in Table 4.

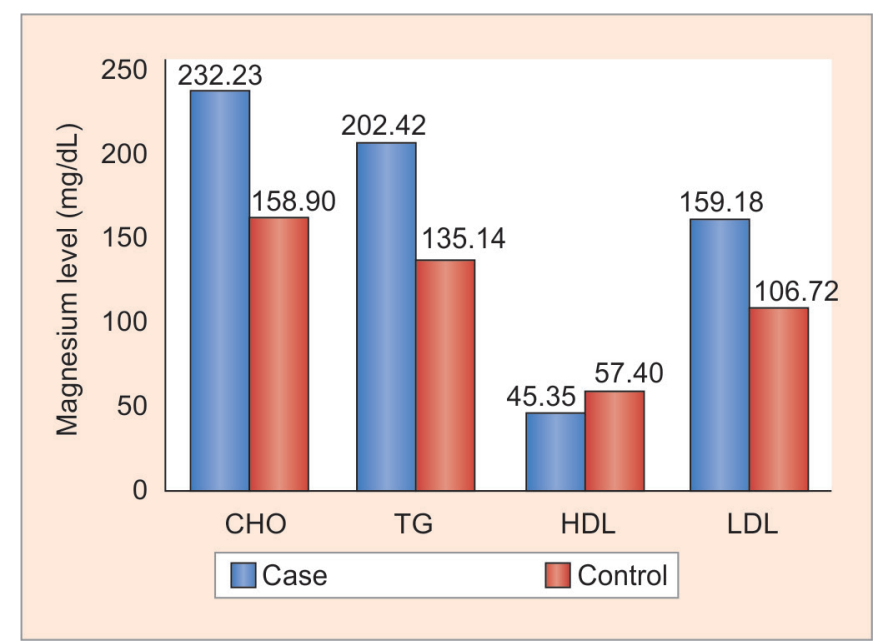

Graph 1: Cholesterol (CHO), TG, HDL-C, and LDL-C level in case and control groups 
Table 2: Cholesterol, TG, HDL-C, LDL-C, FBS, and magnesium level in case and control groups

\begin{tabular}{lllllll}
\hline & $C H O(m g / d L)$ & $T G(m g / d L)$ & $H D L-C ~(m g / d L)$ & $L D L-C ~(m g / d L)$ & $F B S(m g / d L)$ & $M a g n e s i u m(m g / d L)$ \\
\hline Case; $(n=60)$ & & & & & & \\
Mean \pm SD & $232.23 \pm 34.63$ & $202.42 \pm 32.36$ & $45.35 \pm 6.74$ & $159.18 \pm 22.87$ & $194.87 \pm 75.27$ & $1.31 \pm 0.20$ \\
Range & $165-298$ & $142-289$ & $35-61$ & $106-197$ & $99-458$ & $0.94-1.75$ \\
Control; $(n=50)$ & & & & & & \\
Mean \pm SD & $158.90 \pm 33.55$ & $135.14 \pm 30.49$ & $57.40 \pm 6.96$ & $106.72 \pm 15.29$ & $87.78 \pm 9.97$ & $2.02 \pm 0.25$ \\
Range & $95-248$ & $84-214$ & $42-69$ & $82-142$ & $72-106$ & $1.65-2.68$ \\
p-value & $<0.01$ & $<0.01$ & $<0.01$ & $<0.01$ & $<0.01$ & $<0.01$ \\
\hline
\end{tabular}

CHO: Cholesterol; SD: Standard deviation; $p$-value $<0.01$ is considered as significantly different

Table 3: Genderwise comparison of cholesterol, TGs, HDL-C, LDL-C, and magnesium level in case and control groups

\begin{tabular}{lllllll}
\hline & $\mathrm{CHO}(\mathrm{mg} / \mathrm{dL})$ & $T G(\mathrm{mg} / \mathrm{dL})$ & $H D L-C(\mathrm{mg} / \mathrm{dL})$ & $L D L-C(\mathrm{mg} / \mathrm{dL})$ & $F B S(\mathrm{mg} / \mathrm{dL})$ & Magnesium $(\mathrm{mg} / \mathrm{dL})$ \\
\hline Diabetic male & $243.48 \pm 33.30$ & $211.74 \pm 25.16$ & $43.23 \pm 5.79$ & $168.68 \pm 23.01$ & $192.29 \pm 85.81$ & $1.23 \pm 0.20$ \\
Diabetic female & $220.07 \pm 32.54$ & $192.45 \pm 36.46$ & $47.07 \pm 6.55$ & $150.10 \pm 19.22$ & $197.62 \pm 63.41$ & $1.39 \pm 0.16$ \\
p-value & 0.007 & 0.0197 & 0.0191 & 0.0022 & 0.78 & 0.0008 \\
Control male & $165.04 \pm 34.29$ & $133.82 \pm 26.28$ & $59.23 \pm 6.54$ & $107.86 \pm 14.70$ & $90.71 \pm 9.66$ & $1.97 \pm 0.20$ \\
Control female & $162.55 \pm 33.01$ & $136.82 \pm 35.72$ & $54.95 \pm 6.84$ & $105.27 \pm 16.23$ & $84.05 \pm 9.26$ & $2.09 \pm 0.29$ \\
p-value & 0.5015 & 0.7339 & 0.0261 & 0.5584 & 0.0172 & 0.0899 \\
\hline
\end{tabular}

$p$-value $<0.05$ is considered as significantly different

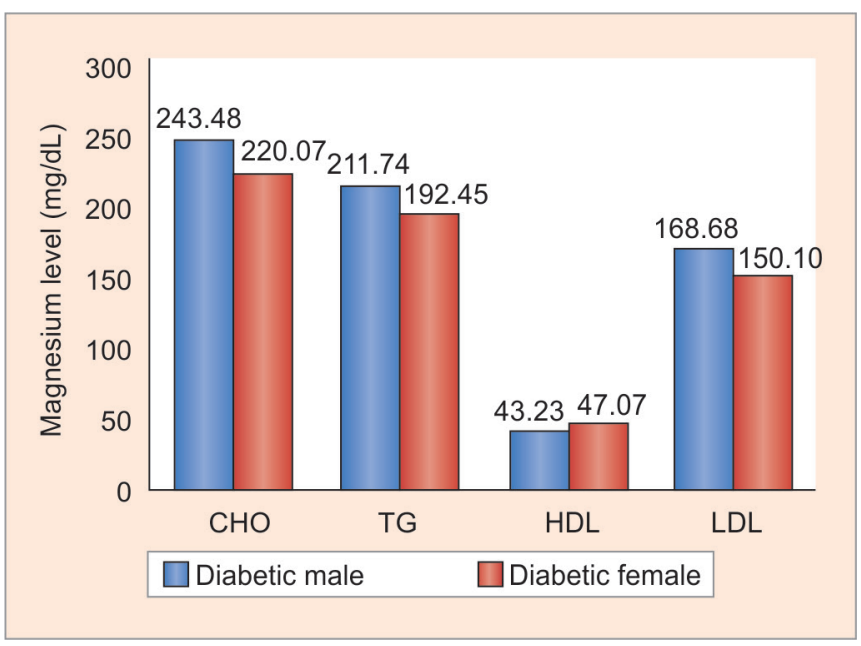

Graph 2: Genderwise comparison of $\mathrm{CHO}$, TG, HDL-C, and LDL-C levels in cases

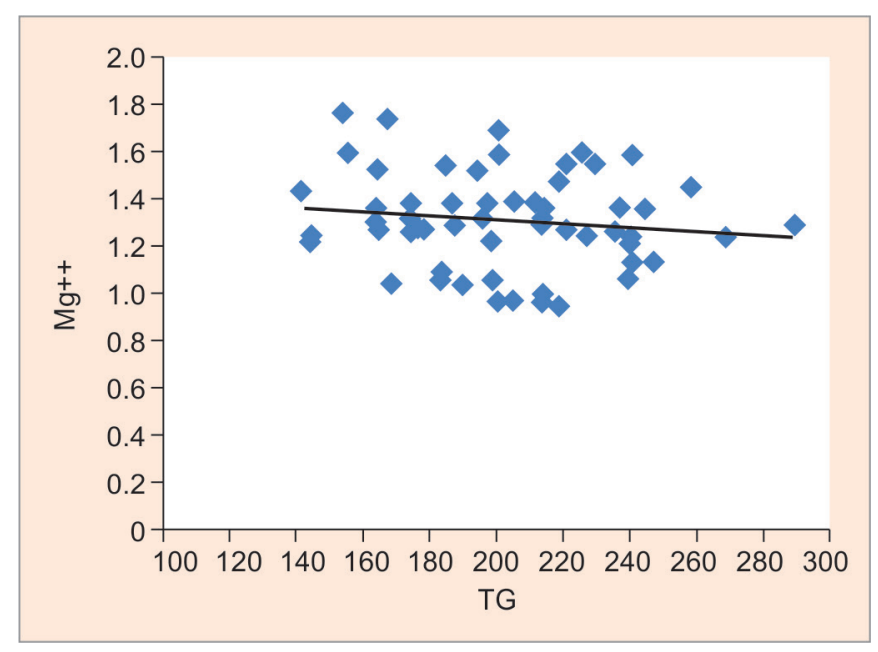

Graph 4: Significant ( $p$-value $<0.05)$ inverse correlation of magnesium $\left(\mathrm{Mg}^{++}\right)$with serum TG $(r$ value -0.14$)$ in diabetic patients

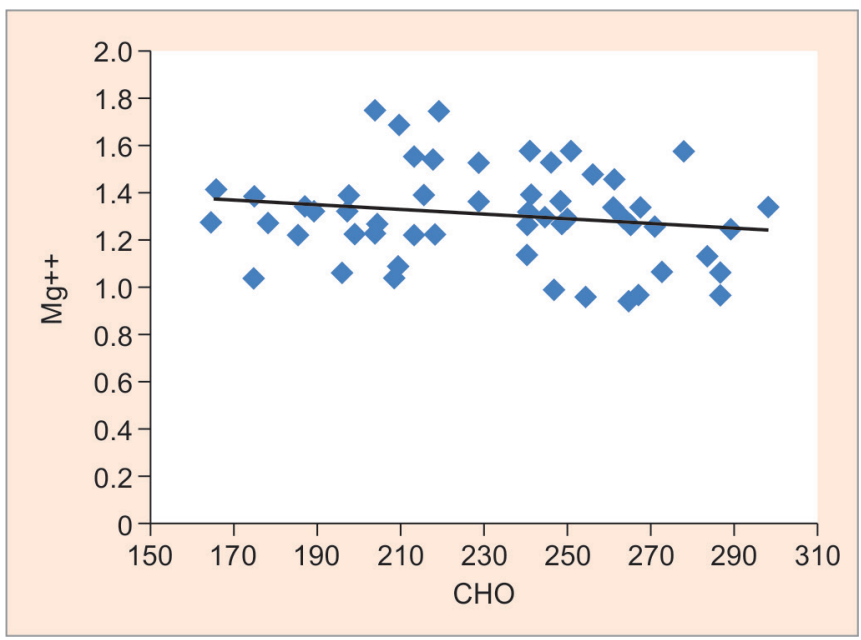

Graph 3: Significant ( $p$-value $<0.05$ ) inverse correlation of magnesium $\left(\mathrm{Mg}^{++}\right)$with serum cholesterol $(\mathrm{CHO} ; \mathrm{r}$ value -0.18$)$ in diabetic patients

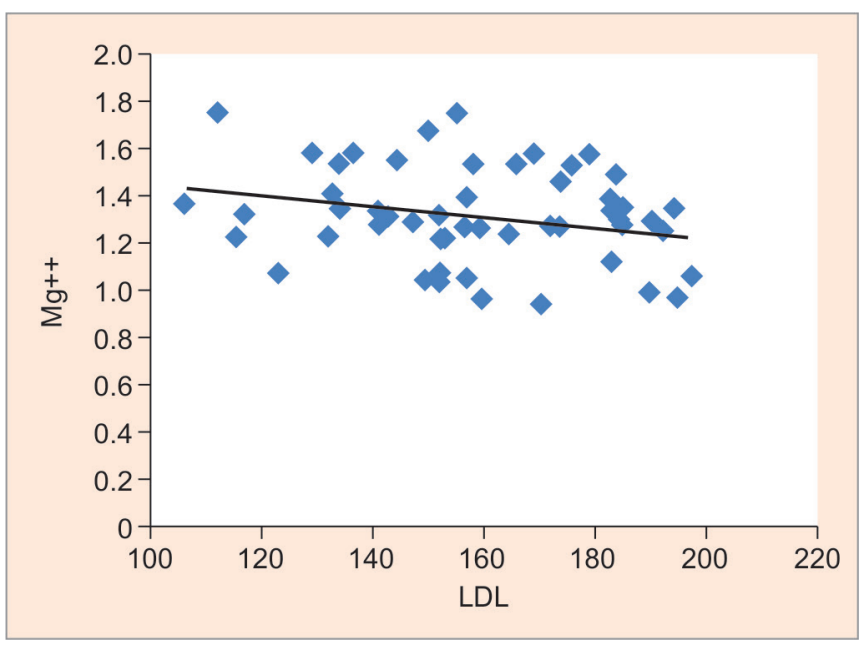

Graph 5: Significant ( $p$-value $<0.05)$ inverse correlation of magnesium $\left(\mathrm{Mg}^{++}\right)$with serum LDL-C ( $r$ value -0.27$)$ in diabetic patients 
Evaluation of Serum Magnesium Level along with Lipid Profile

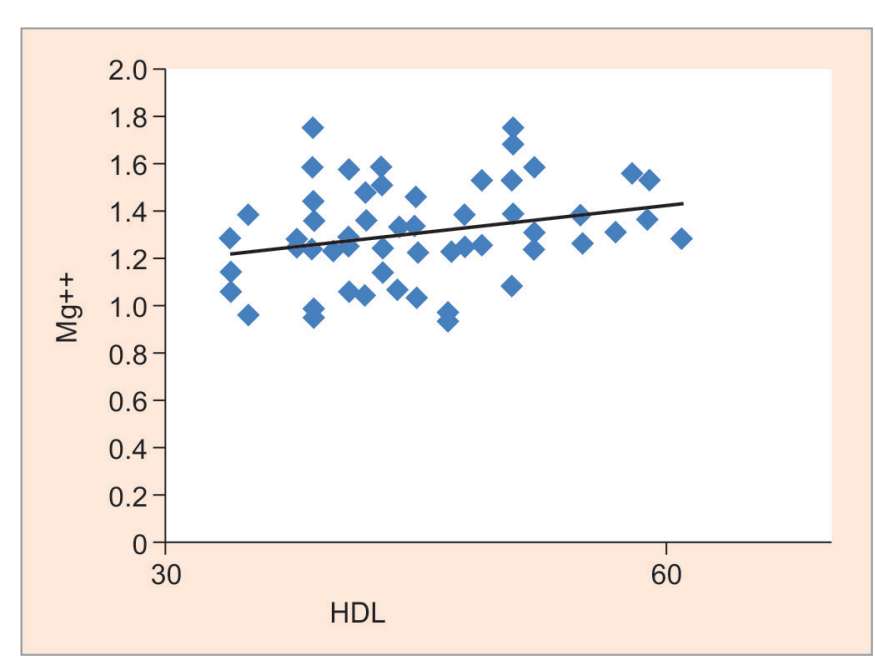

Graph 6: Significant ( $p$-value $<0.05)$ correlation of magnesium $\left(\mathrm{Mg}^{++}\right)$with serum HDL-C (r-value 0.26) in diabetic patients

\section{DISCUSSION}

In the present study, the majority of diabetic patients have higher levels of cholesterol, TGs, LDL-C, and lower level of HDL-C than healthy group. This is consistent with other studies. ${ }^{12-15}$

Accelerated coronary and peripheral vascular atherosclerosis is one of the most common and serious chronic complications of long-term diabetes mellitus. Along with the risk factors, such as hypertension, smoking, obesity, etc., secondary hyperlipidemia has gained significance as an etiologic risk factor for accelerated atherosclerosis. Hyperlipidemia as a metabolic abnormality is frequently associated with diabetes mellitus. Its prevalence is variable, depending on the type and severity of diabetes, glycemic control, nutritional status, age, and other factors. The patients with diabetes have a higher degree of atherosclerosis burden due to dyslipidemia than healthy people. ${ }^{16}$

Diabetes mellitus increases the risk for atherosclerotic vascular disease. There is a twofold to fourfold excess risk of CAD in diabetes mellitus compared with nondiabetic patients. Indeed, 75 to $80 \%$ of adult diabetic patients die of $\mathrm{CAD}$, cerebrovascular disease, peripheral vascular disease, or a combination of these conditions. Patients with diabetes can have many lipid abnormalities, including elevated levels of cholesterol, LDL-C, and TGs; and low HDL-C. Lipid abnormalities may be the result of the unbalanced metabolic state of diabetes (i.e., hyperglycemia and insulin resistance) and improved control of hyperglycemia does moderate diabetes-associated dyslipidemia. However, in diabetics, the LDL-C fraction contains a greater proportion of small dense LDL particles, which are believed to be more atherogenic. ${ }^{17}$

A strong clustering risk factor for $\mathrm{CAD}$ has been observed in diabetic subjects. In diabetes, many factors may affect blood lipid levels. This is because carbohydrates
Table 4: Correlation between magnesium and lipids in control and case groups

\begin{tabular}{lccllc}
\hline & \multicolumn{2}{c}{ Control } & & \multicolumn{2}{c}{ Case } \\
\cline { 2 - 3 } \cline { 5 - 6 } & $\begin{array}{c}\text { Correlation } \\
\text { coefficient } r\end{array}$ & $p$-value & & $\begin{array}{l}\text { Correlation } \\
\text { coefficient } r\end{array}$ & $p$-value \\
\hline CHO & 0.07 & 0.64 & & -0.18 & $<0.05$ \\
TG & -0.12 & 0.39 & & -0.14 & $<0.05$ \\
HDL-C & 0.002 & 0.98 & & 0.26 & $<0.05$ \\
LDL-C & -0.11 & 0.44 & & -0.27 & $<0.05$ \\
FBS & -0.58 & 0.33 & & -0.12 & $<0.05$ \\
\hline P-value & 0.05 & 0.05 &
\end{tabular}

$p$-value $<0.05$ is considered as significantly different

and lipid metabolism are inter-related. If there is any disorder in carbohydrate metabolism, it also leads to a disorder in lipid metabolism. Thus, there is a high concentration of cholesterol and TGs and, due to this, there is a reduction in HDL-C levels. In diabetic subjects, gender plays a significant role on risk of CAD. The diabetic males have moderately higher serum lipid levels as compared with diabetic females. Significant difference in lipid profile of male and female diabetics is because sex hormones play a unique role for lipid metabolism. ${ }^{15}$

Patients with diabetes tend to be dyslipidemic with high TC, TG, LDL-C, and low HDL-C concentrations, which are recognized risk factors for CVDs. Although many factors play roles in the accelerated atherosclerosis observed in diabetes, lipoprotein abnormalities are key contributors. The LDL-C, the main cholesterol-bearing lipoprotein, is a major determinant of atherosclerosis in patients with diabetes. Elevated LDL-C is also associated with an increase in prothrombotic and procoagulant factors. Because of many lipoprotein abnormalities in diabetes, an easily measuring composite indicator may be useful to treat patients with diabetes. Recently, attention has been focused on HDL-C level in the blood. A high HDL-C fraction gives protection against atheroma formation and thus, ischemic heart disease. The HDL-C acts by enhancing the removal of cholesterol from the peripheral tissues and so reduces the body cholesterol pool. ${ }^{12}$

The principal finding of the present study is a significant decrease in serum magnesium levels in the case group, which is correlated with Tripathy et $\mathrm{al}^{18}$ and Rusu et al. ${ }^{19}$ The magnesium depletion is more likely caused by osmotic dieresis, then caused by the indirect hormonal, vitamin C, or ionic effects. Hypomagnesemia represents a risk factor in diabetic retinopathy and tends to decrease the hypoglycemic action of insulin. ${ }^{19}$

In the present study, there is a significant inverse correlation of serum magnesium with cholesterol, TG, and LDL-C. Magnesium deficiency in humans is unlikely to occur from simple lack of food containing these minerals except in advanced forms of malnutrition according to the consensus of a panel on magnesium metabolism in 
diabetes mellitus. Diabetics have an additional risk factor for hypomagnesemia and magnesium status. Magnesium may also play a role in the release of insulin. Magnesium depletion also has an atherogenic potential. The mechanism of long-term complication of diabetes is not clearly explained, and hypomagnesemia may be a contributing factor to these complications, particularly ischemic heart disease, retinopathy, and bone loss. ${ }^{11}$

Usefulness of prolonged magnesium supplementation on reduction of plasma cholesterol and LDL-C and an increase of HDL-C were also shown by Corica et al. ${ }^{20}$ The current study also found inverse correlation of serum $\mathrm{Mg}^{++}$with serum TC, TGs, and LDL-C level, a finding which further supports the importance of magnesium supplementation in diabetic mellitus.

\section{CONCLUSION}

The major highlights of the current study are lower magnesium levels in cases compared with controls. In the current study, the serum magnesium level has been found to be inversely related to cholesterol,TGs, and LDL-C levels. However, it is directly associated with HDL-C level. Hence, it could play a role in controlling the risk of CAD-associated morbidities in future. Therefore, this study suggests the importance of magnesium supplementation in diabetics to prevent the development of its chronic complications, such as CAD. However, separate studies need to be carried out to see the impact of magnesium supplementation on the lipid profile of diabetics in a large population.

\section{REFERENCES}

1. Ramachandran, A.; Das, AK. Diabetology. In: Shah Siddharth N, Paul AM, editors. API textbook of medicine. 7th ed. Mumbai: The Association of Physicians of India; 2003. pp. 1097-1098.

2. American Diabetes Association. Standards of medical care in diabetes. Diabetes Care 2007 Jan;30(Suppl 1):S4-S41.

3. Hu FB, Stampfer MJ, Haffner SM, Solomon CG, Willett WC, Manson JE. Elevated risk of cardiovascular disease prior to clinical diagnosis of type 2 diabetes. Diabetes Care 2002 Jul;25(7):1129-1134.

4. Sridhar RG. Diabetes in India: snapshot of a panorama. Curr Sci 2002 Oct;83(7):1-2.
5. Car J, Patel H, Srishanmuganathan J, Majeed A. Diabetes care in developing countries. Can Med Assoc J 2004 Jan;176(2): 209-212.

6. Khatib NM, Quazi ZS, Gaidhane AM, Waghmare TS, Goyal RC. Risk factors of type 2 diabetes mellitus in rural Wardha: a community based study. Int J Diabetes Dev Ctries 2008 Jul-Sep;28(3):79-82.

7. Mitra A, Bhattacharya D, Roy S. Dietary influence on type 2 diabetes (NIDDM). J Hum Ecol 2007;21(2):139-147.

8. Goldfine AB, Beckman JA. Life and death in Denmark: lesson about diabetes and coronary heart disease. Circulation 2008 Apr;117:1914-1917.

9. Kuussisto J, Mykkänen L, Pyörälä K, Laakso M. NIDDM and its metabolic control predict coronary heart disease in elderly subjects. Diabetes 1994 Aug;43(8):960-967.

10. Sultana R. Impact of duration of type 2 diabetes mellitus on lipid profile. Gomal J Med Sci 2010 Jan-Jun;8(1):57-59.

11. Nasri H, Baradaran HR. Lipid in association with serum magnesium in diabetes mellitus patients. Bratisl Lek Listy 2008;109(7):302-306.

12. Abbasi MA, Hafeezullah, Shah NA, Abro A, Sammo JA. Non high density lipoprotein cholesterol in type 2 diabetes mellitus. Pak J Physiol 2007;3(2):1-4.

13. Bello-Sani F, Bakari AG, Anumah FE. Dyslipidaemia in persons with type 2 diabetes mellitus in Kaduna, Nigeria. Int J Diabetes Metab 2007 Apr;15:9-13.

14. Abou-Seif MA, Youssef AA. Evaluation of some biochemical changes in diabetic patients. Clin Chim Acta 2004 Aug;346(2):161-170.

15. Smith S, Lall AM. A study on lipid profile levels of diabetics and non-diabetics among Naini region of Allahabad, India. Turk J Biochem 2008 Jan;33(4):138-141.

16. Zargar AH, Wandroo FA, Wadhwa MB, Laway BA, MasoodiSR, Shah NA. Serum lipid profile in non-insulin-dependent diabetes mellitus associated with obesity. Int J Diab Dev Countries 1995;15:9-13.

17. Ahmed N, Khan J, Saeed T. Frequency of dyslipidaemia in type 2 diabetes mellitus in patients of hazara division. J Ayub Med Coll Abbottabad 2008 Apr-Jun;20(2):51-54.

18. Tripathy S, Sumathi S, Bhupal Raj G. Minerals nutritional status of type 2 diabetic subjects. Int J Diab Dev Countries 2004;24:27-28.

19. Rusu ML, Marutoiu C, Rusu LD, Marutoiu OF, Hotoleanu C, Poanta L. Testing of magnesium, zinc and copper blood levels in diabetes mellitus patients. Acta Universitatis Cibiniensis Seria F Chemia 2005 Feb;8:61-63.

20. Corica F, Allegra A, Di Benedetto A, Giacobbe MS, Romano G, Cucinotta D, Buemi M, Ceruso D. Effects of oral magnesium supplementation on plasma lipid concentrations in patients with non-insulin-dependent diabetes mellitus. Magnes Res 1994 Mar;7(1):43-47. 